\title{
Localization of intraocular foreign bodies with the low-vacuum contact lens
}

\author{
GOLIN WALKER \\ Southampton Eye Hospital
}

The use of limbal rings sutured to the sclera is a well-established practice in the localizatign of intraocular foreign bodies, and the development of the low-vacuum contact lens, 留 manufactured by the Medical Workshop in Groningen, Holland, obviates the necessity !to attach it to the globe by additional surgery before radiography. The device contains $\vec{\sigma}^{a}$ limbal ring in its substance and a rod in the handle which enables the radiographer $\$$ ensure a true postero-anterior and lateral film.

However, the instructions supplied with the device do not enlarge sufficiently, in view, on precisely how the data derived from these pictures is plotted in order to proviage the information on which the surgery is based.

Being repeatedly aware of the confusion which resulted in this respect with the arfivgl of new resident staff, a model was constructed for lodgement in the theatre which hoped will make all clear for evermore.

\section{Construction}

A square block of wood was cut and the two printed schemas which are supplied with the deve were attached to two adjacent surfaces (Figs I and 2). These were to represent the postero-anterimr and lateral films.

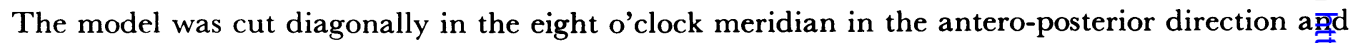
hinged at one edge (Fig. 3). To the diagonal face exposed another printed schema was attachêd

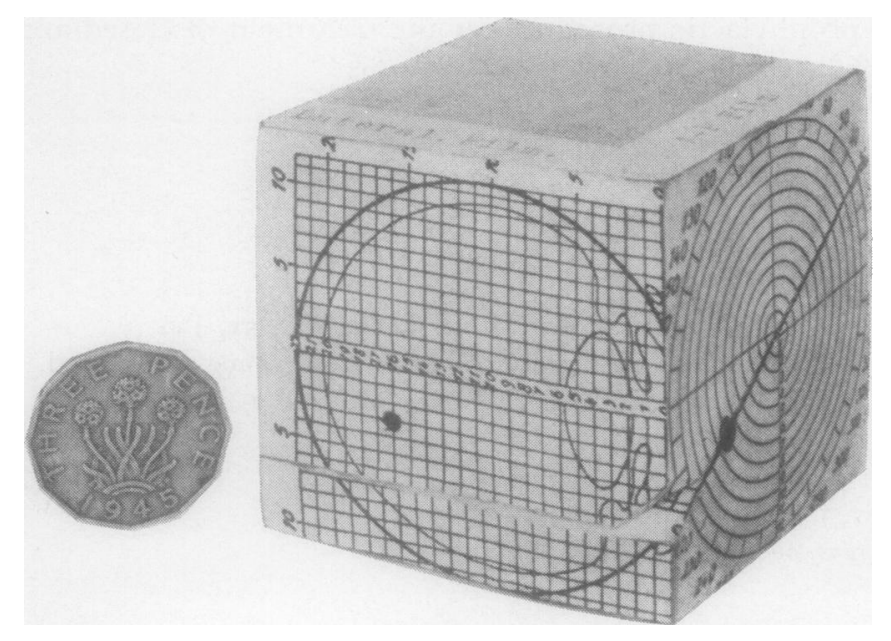

FIG. I Wooden cube showing anterior and lateral views 


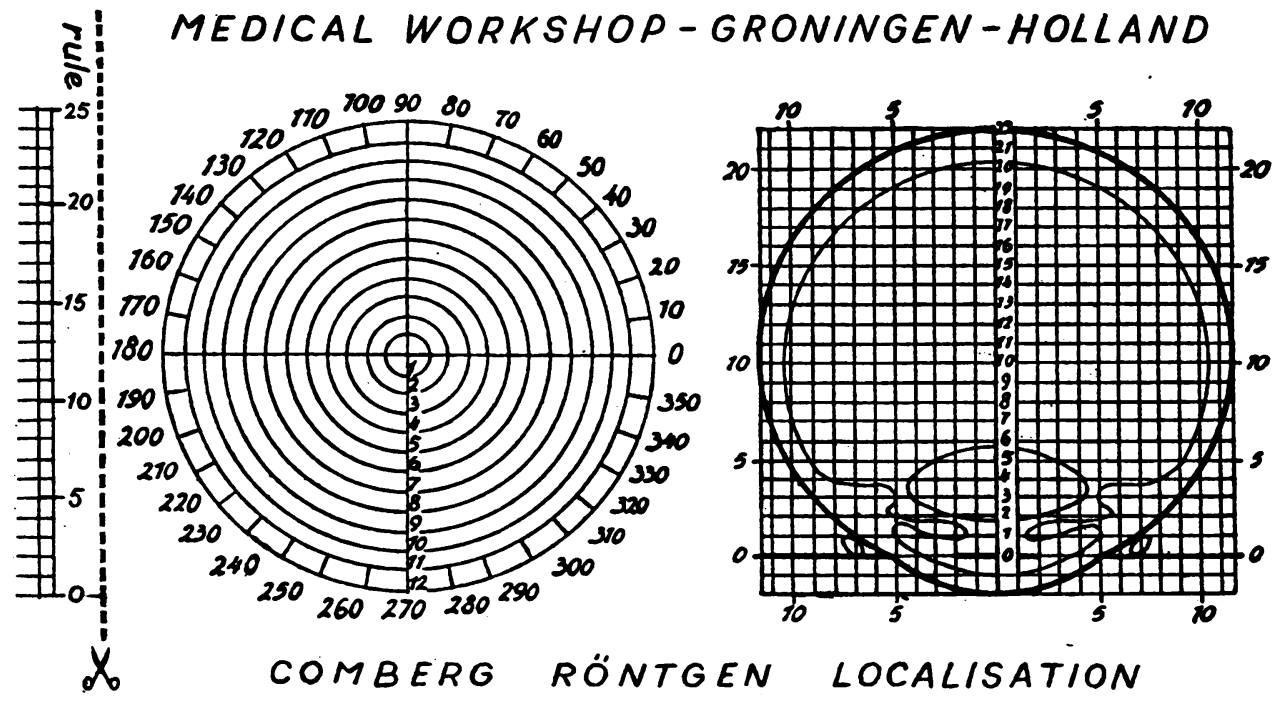

FIG. 2 Printed plans for application to sides of cube

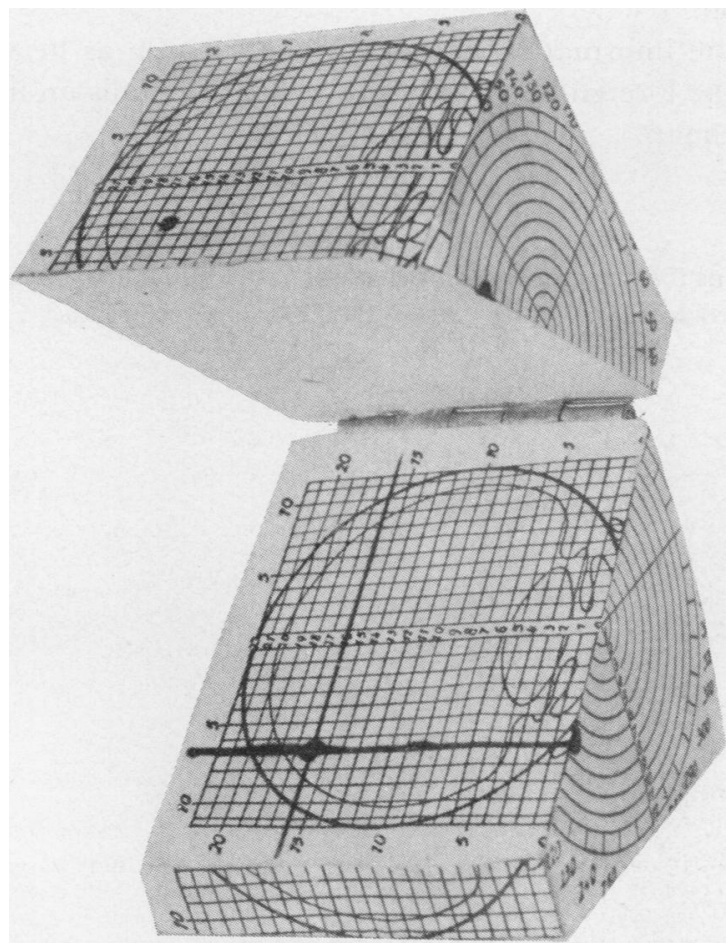

F1G. 3 Hinged cube shown open with foreign body localized from the two exterior views

as shown. A specimen foreign body was marked on the diagonal face and its appropriate position, as it would appear in the radiographs, was marked on the two faces of the outside of the block. Lines were drawn as shown to represent the construction necessary to localize the foreign body. The model demonstrates a foreign body in the 8 o'clock meridian, $7 \mathrm{~mm}$. from the axis of the eye and $16 \mathrm{~mm}$. back from the plane of the limbus. 
It is immediately clear what information is presented and how to obtain it from the radiograpth, the final map being that shown on the diagonal face.

The method supplies a map of an antero-posterior section of the eye containing the axis of eye and the foreign body. It is to be noted that this does not correspond to the lateral face schema unless the foreign body happened to lie in the vertical meridian. On the other hand, the schema $\overrightarrow{f n}$ the front face does correspond with the postero-anterior film.

\section{Method of use}

There are therefore only three pieces of information to be derived from the films :

(r) From the postero-anterior film

A. Plane of the foreign body in clockface or degrees (This will be the plane of the final maj.

B. Distance of the foreign body from the optic axis.

(2) From the lateral film

Distance of the foreign body behind the plane of the limbus as indicated by the limbal riveg.

Any other measurements are inappropriate and illusory.

In practice a plastic grid bearing the two schemas is placed over the two radiographs $\Phi$ obtain these measurements as this allows for the slight magnification produced on the films. Also the printed scale at the edge of the schemas is used if the distance behind the limbids is required, for the same reason.

The method provides just the information needed by the surgeon as he will norma require to operate as near to the foreign body as possible, which entails an incision in the plane represented by the final map.

\section{Reference}

“A Roentgen reference contact lens for foreign body localization”. Medical Workshop, Kranew⿳્心్ 92-94, Groningen, Holland (Brochure) 